\section{Mafias als organisierte Dritte Mafias as organized third parties}

\section{Christopher Dorn / Thomas Hoebel}

\begin{abstract}
:
In the scientific debate on mafias we find neither a common usage nor a shared understanding as to what characterizes a mafia as opposed to other forms of organized crime. To clarify the mafia concept we suggest following Georg Simmel's notion that the appearance of a third party substantively alters the constitution of a social configuration. It is our thesis that mafias act as the third party in a variety of settings by creating or sustaining social orders between at least two other involved parties. In order to acquire and maintain these third party positions, mafias assume the shape of formal organizations. In this light we show the usefulness of this approach through empirical cases that demonstrate how mafias operate as mediators, referees and gatekeepers. The concept "mafias as organized third parties" offers benefits to the study of mafias and organized crime as well as social theory more generally.
\end{abstract}

Christopher Dorn is a lecturer and PhD student in sociology at Bielefeld University as well as a fellow of the Bielefeld Graduate School in History and Sociology (BGHS). His research interests include: sociology of organizations, organized crime, organization and society, social comparison.

E-Mail: christopher.dorn@uni-bielefeld.de

Thomas Hoebel is a research assistant in sociology and PhD student at Bielefeld University as well as a fellow of the Bielefeld Graduate School in History and Sociology (BGHS). His research interests are: sociology of organizations, underground organizations, signaling theory, methodologies of organization research.

E-Mail: thomas.hoebel@uni-bielefeld.de Unangemeldet́ | 85.178.18.244

$$
\text { Heruntergeladen am | 25.10.13 12:32 }
$$

Keywords, dt.: Mafias, Figur des Dritten, Simmel, formale Organisation, Organisierte Kriminalität

Keywords, engl.: mafias, third party, Simmel, formal organization, organized crime

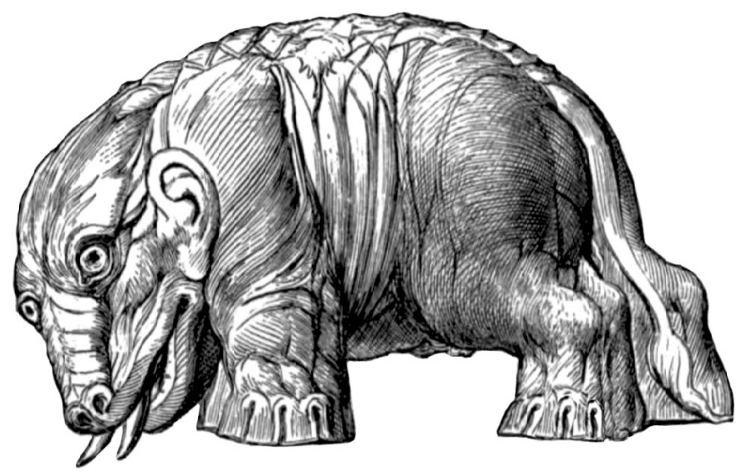


Neben Rotwein, Früchten und Rohöl sind die Begriffe Mafia, Mafioso und mafiös die bedeutenden Exportprodukte Süditaliens.[1] Nicht nur alltagssprachlich und journalistisch sind die Ausdrücke mittlerweile über die Region hinaus schnell bei der Hand, in der Regel um - oftmals effektheischend - fragwürdige Transaktionen zu bezeichnen, in die Politiker und Beamte involviert sind (Lupo 2002, 8). Auch in der sozialwissenschaftlichen Forschung über organisierte Kriminalität haben die Begriffe die Grenzen Siziliens überschritten. Sie haben hier eine Karriere hinter sich, die sich als Entwurzelung begreifen lässt. Denn nachdem die Begriffe historisch zunächst nur mit einem besonderen Verbrechertypus auf Sizilien assoziiert waren, weil die Figurenzeichnung in der 1863 uraufgeführten Komödie „I mafiusi de la Vicaria“ („Die Mafiosi des Gefängnisses von Vicaria“) schnell Nachahmung auf der Straße und in den Amtstuben fand, um lokale Verbrecherbanden zu bezeichnen (Paoli 1999a, 425; Dickie 2007, 86ff.), werden heute kriminelle Organisationen weltweit als Mafias charakterisiert (J. Schneider/P. Schneider 2004, 303). Die sizilianische Cosa Nostra gilt zwar weiterhin als Prototyp der Mafia und ist damit wiederkehrender Bezugspunkt von Analysen organisierter Kriminalität (Paoli 1999a, 9). Längst steht sie aber in einer Reihe mit mehr oder weniger ähnlichen Organisationen, angefangen mit ihren italienischen Nachbarn 'Ndrangheta und Camorra (Savona 2012) bis hin zur US-amerikanischen La Cosa Nostra (Albini 1971), zur japanischen Yakuza (Hill 2006) oder zu den post-sowjetischen Vory v Zakone (Varese 2005), um nur einige Beispiele zu nennen.

Die sozialwissenschaftliche Entwurzelung des Mafiabegriffs aus seinem ursprünglichen Verwendungszusammenhang verlief, stark vereinfachend, in zwei Wellen. Ende der 1960er Jahre wurde Mafia, angestoßen durch die Interpretation der US-amerikanischen „President's Task Force on Organized Crime“ mit dem Soziologen Donald R. Cressey als zentraler Figur, zunächst zum italo-lastigen Synonym für ein aus Sicht der Autoren nahezu bürokratisch organisiertes Verbrechen in den Vereinigten Staaten (Cressey 1969); eine Sichtweise, an der sich die Forschung dann lange kritisch abgearbeitet hat (Schelling 1971; Smith 1976; Haller 1992; instruktiv der Überblick bei Paoli 1999a, 10f.). Infolge der osteuropäischen Systemtransformationen und dem Zusammenbruch der Sowjetunion Anfang der 1990er Jahre wanderte der Mafiabegriff dann in eine weitere Weltregion, um den transnationalen Handel mit illegalen Waren und Diensten zu kennzeichnen (Hessinger 2002; J. Schneider/P. Schneider 2004, 303). Gleichzeitig begründete Diego Gambetta mit seiner am sizilianischen Beispiel gewonnenen und von Thomas Schelling (1971) inspirierten Hypothese, dass
[1] Für wertvolle Kommentare danken wir Axel T. Paul, dem anonymen Gutachter unseres Beitrags, Stefan Kühl, Veronika Tacke und Sven Kette sowie den Teilnehmerinnen und Teilnehmern des Organisationssoziologischen Kolloquiums an der Fakultät für Soziologie der Universität Bielefeld. 
Mafias besondere ökonomische Unternehmungen seien, die privaten Schutz produzieren, anpreisen und verkaufen (Gambetta 1993, 1), ein Forschungsprogramm im Lakatos'schen Sinne (Lakatos 1970), das ohne geografische oder Ethno-Referenzen auskommt. Seine Definition hat sich bisher als ein harter theoretischer Kern erwiesen, der durch zahlreiche Studien verteidigt wurde (Chu 2000; Varese 2005; Hill 2006; Tzvetkova 2008; Varese 2011). Ihre Autoren benötigen prinzipiell keinen inhaltlichen Bezug mehr auf das organisierte Verbrechen in Sizilien, um von Mafia zu sprechen (Gambetta 2011, 6).

Die sukzessive Entwurzelung des Mafiabegriffs zu Zwecken soziologisch-kriminologischer Forschung korrespondiert gleichwohl nicht mit einem einhelligen Begriffsverständnis, auch wenn Gambettas Vorschlag sicherlich einige Prominenz besitzt. Einigkeit besteht nur in zwei Punkten: Mafias sind erstens relativ beständige soziale Ordnungen im Sinn von „going concerns“ (Hughes 1971, 54f.), die sich zumindest für einige Zeit „selbst garantieren“ (um die treffende Formulierung von Heinrich Popitz zu verwenden; Popitz 2010, 14), weil die Interaktion der Beteiligten und die Allokation von Erträgen funktioniert. Zweitens sind Mafias im Kern extra-legale Phänomene (Schelling 1971, 77). Sie operieren jenseits staatlichen Rechts (und seiner supra-staatlichen Äquivalente) und unterhalten dabei gleichzeitig lokalspezifische Beziehungen zu staatlichen Stellen (für Süditalien siehe Paoli 2003, 179ff.).

Während beide Aspekte das genus proximum kennzeichnen, nämlich dass Mafias zur Gattung extra-legaler Ordnungen zu zählen sind, ist ihre differentia specifica strittig. Denn über weitere Merkmale dessen, was als Mafia resp. mafiös bezeichnet werden kann („But what exactly is the mafia?“; Gambetta 1993, 1), besteht erhebliche Uneinigkeit (Gambetta 2011, 6). Theoretisch sind die Begriffe mehrdeutig, insbesondere weil einige Autorinnen und Autoren den Mafiabegriff primär inhaltlich, d.h. über „die Materie der Vergesellschaftung“ (Simmel 1992, 18) spezifizieren und dabei untereinander differieren, andere dagegen eher die Form der Vergesellschaftung in den Vordergrund rücken, „forms that repeated interactions tend to take“ (Martin 2009, 5), hier aber ebenfalls keinen Konsens erzielen.[2]

Inhaltlich finden sich politische, wirtschaftliche und alltagskulturelle Argumente, um die differentia specifica von Mafias zu kennzeichnen (Hessinger 2002, 482f.). Manuel Castells streicht heraus, dass sie nur auf der Basis von Korruption und der Bedrohung staatlicher Funktionäre überleben können (Castells 2010, 210). Gambetta und Peter Reuter sehen demgegenüber keinen empirischen
[2] Wir streben mit der Zusammenstellung der primär inhaltlichen und der eher formalen Argumente, um den Mafiabegriff zu konturieren, keine Vollständigkeit an. Unser Ziel ist allein, seine Mehrdeutigkeit zu verdeutlichen. 
Grund, Mafias über ihren erpresserischen Charakter zu definieren (Gambetta/Reuter 1997, 132f.). Ihre These ist, dass Mafias Kartellvereinbarungen sowohl für legale als auch illegale Waren durchsetzen und absichern und in dieser Hinsicht weniger politisch als vielmehr unternehmerisch sind (Gambetta/Reuter 1997, 116f.). Eine kulturorientierte Argumentationslinie fokussiert dagegen auf die vergleichsweise hohe Gewaltbereitschaft und die familialen Ehrenkodizes von Mafiosi, worin sich gegenüber anderen Bestimmungsversuchen noch stärker der etymologische Ursprung von Mafia resp. mafiös widerspiegelt (Catanzaro 1985, 36; Hessinger 2002, 500ff.).

Formal kreist die Mafiaforschung bis heute im Wesentlichen um die Frage, ob und inwiefern die Mafia organisiert ist, „as a tight-knit sect versus a vague conglomerate of loosely related agents“ (Gambetta 1993, 102); wobei diese ,Formfrage‘ insgesamt enger mit dem sizilianischen Fall verknüpft ist, als es die skizzierten inhaltlichen Bestimmungsversuche sind. Letizia Paoli argumentiert beispielsweise, dass Mafias nicht aufgrund einer (vermeintlichen) inhaltlichen Spezialisierung zu begreifen seien (Paoli 2003, 141ff.), sondern dass das Phänomen Mafia bis heute in der Hauptsache aus zwei Organisationen bestehe, nämlich aus der sizilianischen Cosa Nostra und der südkalabrischen 'Ndrangheta. Beide seien gleichwohl keine streng hierarchisch gegliederten Bürokratien, wie es z.B. Cressey für die ,die Mafia' in den USA behauptete, sondern dezentral und segmentär strukturiert (Paoli 1999a, 13; Paoli 2003, 64). Mark Haller wendet sich ebenfalls gegen die These Cresseys und argumentiert, dass Mafias am ehesten als stabile Verbände brüderlicher Unterstützung zu begreifen seien, ohne dass die in der Regel unternehmerischen Aktivitäten der Mitglieder nennenswert kontrolliert würden (Haller 1992). Er stellt folglich (und vor allem an US-amerikanischen Daten) einen assoziativen Binnencharakter von Mafias heraus, wohingegen z.B. Axel T. Paul und Benjamin Schwalb geltend machen, dass Mitglieder von Mafias als ganze Personen Teil ihres Verbands sind, sie also total inkludiert werden und das gesamte Set ihrer sozialen Beziehungen dem familiären Diktat unterliegt (Paul/Schwalb 2011, 135; siehe dazu auch Pohlmann/Markova 2011, 25). Jane C. und Peter T. Schneider lenken das Augenmerk demgegenüber weniger auf eine organisatorische Binnenstruktur von Mafias als vielmehr auf zwei Vergesellschaftungsformen zwischen Mitgliedern und Nicht-Mitgliedern, die beide in einer quasi-organischen Wechselwirkung mit ihren lokalen Umwelten kulminieren. Einerseits handelt es sich um das Knüpfen dauerhafter Reziprozitätsbeziehungen mit politischen Eliten, andererseits um materielle Versorgungsleistungen für lokale Bevölkerungen (J. C. Schneider/P. T. Schneider 2004, 311ff.). 
Wenn es stimmt, dass wissenschaftliche Begriffe nur dann Kontur gewinnen, wenn sie ihren Geltungsbereich nachvollziehbar einschränken (Luhmann 1992, 124), dann ist es um den Mafiabegriff und seine Ableitungen schlecht bestellt. Insbesondere ist offen, was Mafias eigentlich soziologisch von anderen kriminellen Organisationen unterscheidet. In der Literatur zeichnen sich vor allem zwei Probleme ab, die seiner weiteren Konturierung entgegenstehen. Jedes kontraktive Verständnis, z.B. Gambettas Vorschlag, dass Mafias privaten Schutz verkaufen, setzt sich dem Vorwurf der Einseitigkeit aus (Paoli 2003, 143). Kontraktion bietet allerdings den Vorteil, den Begriff generalisiert und weltweit zur Identifikation und Erklärung zahlreicher Fälle verwenden zu können, in denen extra-legale Instanzen z.B. auf unternehmerische Weise quasi-staatliche Ordnungsfunktionen übernehmen (Gambetta 2011, 6). Jede expansive Bestimmung, z.B. Philipp Hessingers an Marcel Mauss` Terminus angelehnte These, die Mafia sei ein totales soziales Phänomen, ,in dem alle Arten von Institutionen gleichzeitig und mit einem Schlag zum Ausdruck kommen“ (Hessinger 2002, 484), wird zwar der inhaltlichen und formalen Komplexität einer sozialen Erscheinung gerecht. Der Begriff ist dann aber in letzter Konsequenz nur auf wenige Fälle anwendbar, die sich in ihrer „Verschlingung“ (Simmel 1992, 30) von individuellen Existenzen, Sozialformen und Inhalten der Vergesellschaftung einigermaßen ähneln. Letztlich steht die soziologische Forschung vor der Alternative, ob sie weiter an der Entwurzelung des Mafiabegriffs festhält, um ihn zur Erklärung einer Äquivalenzklasse extra-legaler Ordnungen zu nutzen, oder ob sie diese theoretische Ambition aufgibt und ihn nur wissenssoziologisch behandelt, weil er in der gesellschaftlichen Kommunikation zur Selbstbeschreibung eingesetzt wird, z.B. in bestimmten Weltregionen oder journalistisch.

Mithilfe eines klassischen Arguments von Georg Simmel optieren wir dafür, an seiner Entwurzelung festzuhalten. In seiner mittlerweile klassischen Studie „Die quantitative Bestimmtheit der Gruppe“, dem zweiten Kapitel seiner erstmals 1908 erschienenen „großen Soziologie“, erörtert Simmel, dass bestimmte Formen der Vergesellschaftung eine Mindestzahl an Beteiligten voraussetzen, nämlich drei. Er macht dabei geltend, dass der Dritte gegenüber dem Zweiten einerseits und dem Vierten (oder mehreren) andererseits eine besondere qualitative Bedeutung für die Entstehung und Erneuerung sozialer Ordnungen hat (Ziemann 2011, 211). Erstens entwickeln sich einigermaßen dauerhafte Verhältnisse zwischen zwei Elementen oftmals erst durch einen Dritten (Simmel 1992, 114), sei es in Eintracht oder in Zwietracht (Freund 1976, 91). Zweitens modifiziert das Hinzutreten von Vierten, Fünften oder Sechsten den Charakter einer bestehenden Konfiguration nicht in gleicher Weise, wie es ein Dritter schafft (Simmel 1992, 117f.). 
In einem an Simmels Studie angelehnten Perspektivwechsel auf die „bloße Zahl“ (Simmel 1992, 63) sehen wir eine in der bisherigen Mafiaforschung nicht explizit ausgeschöpfte Option, um zu präzisieren, was Mafia „exactly is“, wenngleich implizit häufig mit quantitativen Argumenten gearbeitet wird, sofern extra-legale Konfigurationen unter Beteiligung von Mafias beschrieben werden. Unsere These ist, dass der Begriff der Mafia empirisch einen Dritten bezeichnet, der ein besonderer, nämlich ein organisierter Dritter ist, der das Problem der Anerkennung von Ansprüchen zwischen zwei oder mehr Parteien bearbeitet. Wir argumentieren somit, dass er nur solche kriminellen Organisationen bezeichnet, die (a) wiederkehrend als Dritter in Erscheinung treten, damit (b) die Entstehung und Erneuerung einer sozialen Ordnung ermöglichen und (c) diese Position für eine bestimmte Dauer aktiv behaupten können. Darin sehen wir gleichsam die Option, die vielfältigen empirischen Beziehungen zwischen Mafias und staatlichen Stellen zu systematisieren. Diese Option können wir an dieser Stelle nur andeuten, weil wir uns in diesem Beitrag zunächst auf das numerische Argument über die differentia specifica von Mafias konzentrieren möchten. Unsere Idee ist, je nach historischer und lokaler Situation danach zu fragen, ob und inwiefern staatliche Stellen ein Element in mafiösen Konfigurationen sind. Theoretisch sind drei numerische Positionen denkbar: (a) ganze Behörden oder einzelne Beamte sind als Einer oder Anderer involviert, (b) staatliche Stellen fungieren selbst als mafiöse Dritte bzw. Mafias fungieren als Behörden oder (c) der Staat steht als Vierter der Mafia gegenüber, z.B. als Konkurrent oder als Komplementär (siehe dazu wiederum für Süditalien Paoli 2003, 179ff.).

\section{Mafias als Elemente in extra-legalen Konfigurationen}

Die soziologische Forschung verdankt Georg Simmel die basale Einsicht, dass sich zahlreiche soziale Formen bereits als Konfiguration aus Zweien realisieren, die meisten jedoch mindestens ein drittes „Element“ erfordern.[3] Typische Zweierkonfigurationen, die keinen Dritten benötigen oder ihn sogar notwendigerweise ausschließen, sind z.B. der Tausch, der Kampf, die Kooperation und die Intimbeziehung. Die Vermittlung, die Übersetzung, die Rivalität oder die Intrige sind demgegenüber Beispiele für Dreierkonfigurationen, sie setzen also mindestens drei Elemente zwingend voraus (für eine umfangreichere Liste mit entsprechenden Literaturreferenzen siehe Fischer 2008, 124f.).

Jede historisch-konkrete Konfiguration ist in dieser Sicht ein Produkt der spezifischen Wechsel-
[3] Anstelle des Begriffs der Gruppe, der nach dem heute erreichten Stand soziologischer Theorie irreführend ist, weil er von Simmel zu expansiv angesetzt ist, sprechen wir konsequent von Konfigurationen. Es handelt sich dabei um einen alternativen Begriff, den Simmel selbst immer wieder nutzt, um allgemein von Formen der Vergesellschaftung zu sprechen, ohne bereits ihren Inhalt spezifizieren zu müssen. Konfigurationen sind in dieser Sicht also nicht durch ein Gefühl der Zusammengehörigkeit bestimmt, wie es für soziale Gruppen gilt (Tyrell 2008). 
wirkungen zwischen einer Mindestzahl an Elementen, womit Simmel in der Regel natürliche Personen meint (wenngleich wir mit unserer Begriffswahl die u.a. von Harrison White gezogene Option gewinnen, theoretisch von natürlichen Personen abstrahieren zu können und andere „Identitäten“ als Element zu begreifen; White 2008). Element ist eine Person (oder ihr Äquivalent) gleichsam nicht außerhalb einer Konfiguration, die es mit erzeugt. Es ist vielmehr selbst durch seine Wechselwirkung mit entweder einem oder zwei anderen Elementen bestimmt und folglich an eine empirische Konfiguration gebunden (Simmel 1992, 151).[4]

Gegenüber einer primär inhaltlichen oder primär formalen Bestimmung des Mafiabegriffs gewinnen wir mit numerischen Argumenten eine zusätzliche Möglichkeit, um ihn theoretisch zu konturieren. Der Ausgangspunkt für diesen gedanklichen Umweg ist die zugegeben etwas künstlich anmutende Frage, ob und inwiefern Mafias resp. Mafiosi sich elementar in dem oben skizzierten Sinne begreifen lassen. Konstituieren sie sich also typischerweise als ein Element, A oder B, in Zweierkonfigurationen? Oder gewinnen sie ihr Profil im Wesentlichen in Dreierkonfigurationen, also als A, B oder C? Und in welchen Wechselwirkungen stehen sie dann jeweils mit ihren Gegenübern?

Rein logisch gesehen sind von diesem Ausgangspunkt aus - und verbunden mit dem einhellig geteilten Argument, dass Mafias im Kern extra-legal sind (Schelling 1971) -, drei Annahmen möglich, die sich gegenseitig ausschließen, um wahr sein zu können:

1. Mafias sind zwingend eine Partei in extra-legalen Zweierkonfigurationen, aber nicht notwendigerweise auch in Dreierkonfigurationen.[5]

2. Mafias partizipieren zwingend in extra-legalen Dreierkonfigurationen, sind aber nicht notwendigerweise auch Element in Zweierkonfigurationen.

3. Mafias sind notwendigerweise Partei in extra-legalen Zweier- und in Dreierkonfigurationen.

Mit unserer Überlegung, Mafias seien organisierte Dritte, neigen wir dabei der zweiten Annahme zu bzw. gehen sogar noch darüber hinaus, indem wir behaupten, dass Mafias nicht einfach nur eine Partei in Dreierkonfigurationen sind, sondern hier als Dritter fungieren, durch den zwei andere Elemente erst zusammenfinden - und zusammenbleiben. Mafias sorgen, so unsere These, für die Anerkennung von Ansprüchen zwischen zwei oder mehr Parteien, und zwar (a) indem sich die Mafiosi formal organisieren, (b) im Zweifel gewaltsam vorgehen und (c) für diese Leistung kassieren.
[4] Nicht zu verwechseln ist damit freilich, dass die Voraussetzungen, überhaupt Element sein, also als Anderer für den Einen oder als Dritter für die Beiden fungieren zu können, in der Regel außerhalb einer Konfiguration liegen (Simmel 1992, 42ff.).

[5] Der Begriff der Partei anstelle des Ausdrucks Element ist alltagssprachlich geläufiger. Um die Lesbarkeit zu erhöhen, verwenden wir daher beide im weiteren Verlauf synonym. 


\section{Mafias als Dritte}

Schutz, Korruption, Organisation von Kartellen, Gewaltausübung, Versorgung - die Liste vornehmlich extra-legaler Konfigurationen unter Beteiligung von kriminellen Organisationen, die wir allein aus der einleitenden Skizze des Forschungsstands destillieren können, ist lang. Die These, dass das Distinktionsmerkmal von Mafias gegenüber anderen kriminellen Organisationen in der voraussetzungsvollen Besorgung von Anerkennung liegt, erscheint daher vermutlich einigermaßen überraschend. Letztlich ist sie das Ergebnis einer numerisch ansetzenden Lektüre von Kritiken am Gambetta'schen Forschungsprogramm, Mafias über eine private Schutzfunktion zu fassen. Diese Kritiken laufen im Kern auf zwei Punkte hinaus, die besonders prägnant bei Paoli zu finden sind. Erstens argumentiere Gambetta (und mit ihm diejenigen, die sich an seine Thesen anlehnen) funktionalistisch, indem er die Existenz von Mafias ahistorisch aus ihrer (vermeintlichen) Rolle ableite, die sie in manchen Regionen gesellschaftlich spielen, und nicht hinterfrage, dass sie gewaltsam und keinesfalls alternativlos in diese Position gerückt sind (Paoli 2003, 143). Zweitens wird Gambetta angegriffen, weil er die schiere Erpressung von Schutzgeld als einer, numerisch reformuliert, Zweierkonfiguration zu einer Dreierkonfiguration umdeute, in der Mafias privaten Schutz für die Aktivitäten von zwei weiteren Parteien produzieren (Paoli 2003, 159f.). Während also Paoli argumentiert, dass Mafias Schutzgeld kassierten, um die Erpressten vor sich selbst, gemeint sind die Mafiosi, zu schützen, verneinen die Forscher um Gambetta zwar nicht, dass sich Mafias in Erpressungen engagieren. Sie sehen darin aber nicht ihr zentrales Merkmal, sondern in „genuine services like protection against extortion; protection against theft and police harassment; protection for thieves; protection in relation to informally obtained credit and the retrieval of loans; the elimination of competitors; the intimidation of customers, workers, and trade unionists for the benefit of employers; the intimidation of lawful right holders; and the settlement of a variety of disputes" (Varese 2011, $5 \mathrm{f}$.). Mafias mögen diese Leistungen schlecht ausführen, gibt Gambetta selbst zu bedenken. Doch sie machen es (Gambetta 2009, 47), und zwar, so unsere Simmel'sche Ergänzung, konsequent als Dritte.

Der erste Kritikpunkt ist sicherlich - soweit wir die einschlägige Literatur überblicken - berechtigt. Instruktiv ist z.B. Mike Dashs Studie über die Etablierung der „ersten Familie“ in New York, die Morellos in Little Italy. Er belegt eindrücklich, dass dieser Clan - entgegen allen Mythen, die sie als Verteidiger des armen Volkes oder als Ehrenmänner stilisierten - zumindest bis zur Prohibition 
in den 1920er Jahren ein Parasit war, der andere Italiener mit Gewalt zu einer Tributpflicht zwang (Dash 2010, 187f.). Jüngere Anti-Mafia-Bewegungen in Süditalien mit Vereinen wie addiopizzo und libero futuro als Kristallisationspunkten belegen zudem die historische Kontingenz von Mafias (Superti 2009), wenngleich ihr Erfolg gegenwärtig noch offen ist (Vaccaro 2012).

Der zweite Kritikpunkt ist ebenfalls berechtigt, sofern er aufdeckt, wie problematisch der theoretische Status des in der Mafiaforschung prominenten Schutzbegriffs ist. Handelt es sich eigentlich um Schutz, den Mafias gewähren? Der Ausdruck erscheint reichlich euphemistisch, wenn man wie Paoli geltend macht, dass die Initiative, Schutz zu produzieren, in der Regel bei gewaltbereiten Kriminellen liegt und nicht bei den ,Konsumenten', die sich durch Andere bedroht sehen und sich deswegen an die lokalen Mafias wenden.

Gleichzeitig aber läuft die zweite Kritik ein Stück weit ins Leere. Denn der Schutzbegriff des Gambetta'schen Forschungsprogramms abstrahiert davon, wer eigentlich initiativ wird, um Schutz zu produzieren resp. zu konsumieren. Damit verbunden liegt die Stärke der Studien, die einen entsprechend ,entlasteten` Begriff zugrunde legen, vielmehr darin, dass ihre Autoren zahlreiche Dreierkonfigurationen identifizieren und beschreiben, die nur unter Beteiligung von denjenigen, die sie als Mafiosi kennzeichnen, bestehen können. Zwei oder mehr konkrete Elemente sind, formalsoziologisch reformuliert (Simmel 1992, 114), also überhaupt erst - oder zusätzlich zu einer bestehenden Beziehung - durch Mafias miteinander verbunden. Viele dieser Konfigurationen lassen sich aber nur schwerlich als Schutzbeziehung begreifen, wie z.B. Federico Vareses Liste von Phänomenen, die unter den Schutzbegriff gebucht werden können, mit Formulierungen wie „the intimidation of customers, workers, and trade unionists for the benefit of employers; the intimidation of lawful right holders; and the settlement of a variety of disputes" (siehe oben) erahnen lässt. Aus unserer Sicht ist daher nicht die Erpressung (Paoli) oder Produktion (Gambetta) von Schutz das primäre Merkmal, das Mafias begrifflich bestimmt, sondern dass sie in vielfältiger Art und Weise Dritte sind und soziale Ordnungen zwischen mindestens Zweien erzwingen.[6] In einer konsequent numerischen Sicht sehen wir die Chance, die bei Gambetta, Varese, Hill u.a. manchmal etwas gezwungen wirkende Reduktion empirischer Daten auf die Schutzthematik (P. Schneider 1994; Nelken 1995, 287) zugunsten eines generalisierten Mafiabegriffs zu überwinden, der den zentralen Einsichten ihrer Studien weiterhin gerecht wird und sich gleichzeitig als anschlussfähig an Arbeiten ,jenseits' des Gambetta'schen Forschungsprogramms erweist. Schutz ist in dieser Sicht nur eine von vielen mögli-
[6] Unbestritten sind Mafias auch Elemente in Zweierkonfigurationen, z.B. als Geschäftsleute im Bausektor oder in der Raffinierung und im Vertrieb von Heroin (Chubb 1996, 280). Insbesondere die Mitglieder der Cosa Nostra genieBen hohe Freiheitsgrade, selbst geschäftlich aktiv zu werden (Paoli 2003, 147), wenngleich Gambetta geltend macht, dass Mafiosi sich in anderen Dingen als „enforcement jobs“ generell eher als inkompetent erweisen (Gambetta 2009, 48). Nur der Eine oder der Andere von Zweien zu sein, ist aber eben nicht die Besonderheit von Mafias gegenüber anderen kriminellen Organisationen, sondern ihre diversen Positionen als Dritte. 
chen Drittenpositionen von Mafias, im Rahmen derer sie soziale Ordnungen erzwingen. Der Vorteil einer primär numerischen Sicht ist somit, dass wir inhaltlich offener werden, Mafias zu begreifen - insofern wir nachweisen können, dass sie als Dritte Konstitutionsbedingung unwahrscheinlicher sozialer Ordnungen sind.

In der heute verfügbaren historiografischen, anthropologischen und soziologischen Literatur zeichnen sich über unterschiedliche Epochen und nationalstaatliche Kontexte hinweg gesehen mindestens drei typische Drittenpositionen von Mafias ab: als Vermittler, als Unparteiische und als „Torwächter“.[7] Wir sind uns sicher, dass sich die Liste darin nicht erschöpft, beschränken uns aber auf diese drei Typen, um das Potential der numerischen Perspektive zur Konturierung des Mafiabegriffs deutlich zu machen.[8]

Mafias als Vermittler - Ein klassisches Beispiel für die Einnahme der Position des tertius mediator (Simmel 1992, 125f.) durch Mafias ist die Vermittlung zwischen Politikern und einfachen Bürgern, wie sie für Süditalien, besonders Kalabrien und Sizilien, gut dokumentiert ist. Mit der ab 1882 beginnenden Ausweitung des italienischen Wahlrechts in Richtung eines allgemeinen Zugangs ergab sich für die verschiedenen Mafias eine Möglichkeit, als Dritter zu fungieren, da die politischen Eliten zunehmend auch auf Wählerstimmen aus der Mittel- und Unterschicht angewiesen waren. So nutzten Mafiosi ihren bereits vorhandenen Einfluss auf lokale Bevölkerungen (durch Gewaltandrohung, Schutzgeld etc.), um Wählerstimmen zu lenken und Gegenkandidaten einzuschüchtern.[9] Für die Beschaffung von Wählerstimmen erhielten die Mafiosi im Gegenzug von den Politikern diverse Vergünstigungen, unter anderem Nachsicht bei der Strafverfolgung, die sie wiederum nutzen konnten, um ihre Position gegenüber der Bevölkerung zu stärken, etwa durch die Vergabe von Arbeitsplätzen (Paoli 1999b, 22; P. Schneider/J. Schneider 2003, 118f.).

In den USA realisierten sich seit Beginn des 20. Jahrhunderts vergleichbare Dreierkonfigurationen im Rahmen sogenannter labor rackets. Zentral ist dafür die Beeinflussung und Infiltration von Gewerkschaften. Dabei macht sich die jeweilige Mafia die gegenseitige Abhängigkeit von Arbeitgebern und Arbeitnehmern zunutze und spielt diese gegeneinander aus. Anschaulich wird dies am Fall eines labor rackets der New Yorker Mafia, die später unter dem Namen Gambino-Familie bekannt geworden ist, im Hafengebiet von Brooklyn. Die dortigen Hafenarbeiter mussten der Mafia gegenüber regelmäßig eine Summe entrichten, um überhaupt Beschäftigung zu finden. Wer sich dagegen sträubte, fand bei der International Longshoremen's Association aufgrund des Einflusses der Mafia
[7] Zeitlich erstrecken sich die Daten etwa von der Mitte des 19. bis zum Ende des 20. Jahrhunderts, geografisch auf Italien, Japan und die USA. Wir schließen damit gleichwohl keine Epochen oder Regionen aus unserer Argumentation aus. Vielmehr ist unsere Einschränkung ein Ausdruck der aktuellen Datenlage, die eine Schlagseite in Richtung der genannten Länder hat, weil hier die wohl intensivste Forschung zu Formen organisierter Kriminalität existiert, in Italien nicht zuletzt befördert durch empirische Einsichten im Rahmen zahlreicher Ermittlungserfolge und Gerichtsprozesse gegen Mafiosi seit den 1980er Jahren (Paoli 2003, 13).

[8] Da die drei beschriebenen Drittenpositionen induktiv gewonnen sind, bilden sie ebenfalls keine erschöpfende Typologie (siehe zu einem solchen Missverständnis in Bezug auf die von Simmel beschriebenen tertius mediator, tertius gaudens und tertius imperator den Hinweis von Hanoch Tennen in Freund 1976, 103).

[9] Eine detaillierte Darstellung dieser Vorgehensweise, die zeigt, wie die Bürger zur Stimmenabgabe für den von der Mafia propagierten Kandidaten gebracht wurden, findet sich z.B. in Anton Bloks Langzeitstudie über ein sizilianisches Dorf (Blok 1981, 205ff.). 
kein Gehör und musste, wenn er die Polizei informierte, mit dem Tod rechnen. Aufgrund dieser Position gegenüber den Arbeitern konnte wiederum Geld von den Reedereien gefordert werden, da andernfalls ihre Schiffe nicht gelöscht wurden und es zu Streiks und Sabotage kam (Davis 1994, 52f.).[10]

Ein weiterer Beleg findet sich im Kontext der japanischen Bauindustrie. Aufgrund der dort herrschenden Arbeitsbedingungen besteht ein Großteil der Arbeitskräfte aus ungelernten Tagelöhnern. Für diese Arbeiter existiert eine staatliche Vermittlungsagentur, die tatsächlich aber nur etwa 20 bis 30 Prozent der Vermittlungen tätigt. Der Großteil geht auf Arbeitsvermittler (tehaishi) zurück, die überwiegend der Yakuza angehören oder wenigstens dauerhafte Beziehungen zu ihr unterhalten. Sie intensivieren die Verbindung zwischen den Arbeitgebern und den Arbeitnehmern insofern, als die Inanspruchnahme dieses Dienstes sich für beide Seiten nutzbringender gestaltet, als wenn die staatliche Agentur eingeschaltet ist. Für die Arbeiter ist die Beteiligung der tehaishi vorteilhaft, weil ihre Löhne, trotz des Anteils, den der Vermittler von ihnen fordert, immer noch über denen von staatlich vermittelten Arbeitern liegen. Im Gegenzug erhalten die Arbeitgeber ein stärker vorselektiertes Personal, als dies bei den staatlichen Stellen der Fall ist, und garantiert konfliktfreie Beziehungen zu den Beschäftigten (Hill 2006, 112f.).

Ein letztes Beispiel für Mafias als tertius mediator: Im Zigarettenschmuggel treten neapolitanische Camorristi als Vermittler zwischen Produzenten und Konsumenten auf und stärken die Bindung zwischen diesen. Dabei wurden die Zigaretten für den illegalen Handel nicht mehr gestohlen, sondern direkt von den großen Tabakkonzernen akquiriert, die zu diesem Zweck ein paralleles Exportsystem betrieben. Den Konsumenten wurde dadurch ermöglicht, Zigaretten bekannter Marken ohne Steueraufschlag zu erwerben (Behan 2009, 176f.).

Mafias als Richter - Eine zweite Drittenposition von Mafias ist die des richtenden Unparteiischen in Konflikten zwischen Zweien (Simmel 1992, 126ff.). Mafiosi haben diese Stellung typischerweise im Falle von Ehrverletzungen weiblicher Personen eingenommen, insbesondere bei geschlechtlichen Beziehungen zu unverheirateten Frauen.[11] Sie zwingen den männlichen ,Verursacher` zu einer Heirat, die als Wiederherstellung der Ehre gilt (Blok 1981, 312ff.; Paoli 2003, 163). Bekannt ist z.B. eine Intervention des lokalen 'Ndrangheta-Clans in Corigliano. Hier wurde ein minderjähriges Mädchen schwanger, weshalb der Vater des Mädchens auf eine Hochzeit mit dem Vater des Kindes pochte. Daraufhin setzte sich der Liebhaber nach Argentinien ab. Durch das Eingreifen des
[10] Im New Yorker Baugewerbe findet sich das gleiche Muster (Davis 1994, 227f.)

[11] Zum mediterranen Ehrkomplex siehe Giordano 1994. 
Clans kehrte der Bräutigam in spe zurück und die Vermählung konnte stattfinden. Dabei lösten die 'Ndranghetisti auch das Problem, einen geeigneten Priester zu finden, der bereit war, eine Minderjährige zu trauen (Dietz 2011, 169).

Die japanischen Yakuza treten ebenfalls in einer Art und Weise auf, die sich eher als Mediation denn als Schutz bezeichnen lässt. Sie werden z.B. von an Verkehrsunfällen Beteiligten zu Rate gezogen, ein Phänomen, das keine Seltenheit darstellt, weil die rechtlichen Verfahren, um Versicherungsansprüche geltend zu machen, sehr aufwendig sind. Gegen einen Anteil an der ausgezahlten Schadenssumme üben die Yakuza Druck auf die jeweilige Versicherung aus, was den Vorgang in der Regel beschleunigt. Ähnlich verhält es sich im Bereich der Eintreibung von Schulden, die in Japan nur Anwälte oder einige wenige lizenzierte Firmen durchführen dürfen. Da auch dieser Vorgang sehr kosten- und zeitträchtig ist, wenden sich die Gläubiger an die Yakuza, die unter Zuhilfenahme von Drohungen und Gewalt gegen einen Prozentsatz der Schuldsumme für deren Rückzahlung sorgen. Auch im Bereich der Insolvenzabwicklung werden die Yakuza auf diese Weise tätig (Hill 2006, 119ff.).

Ein weiteres Beispiel stellt die Vermittlung zwischen Dieben bzw. Räubern und den Geschädigten dar, die auf zwei Arten erfolgen kann. Zum einen findet tatsächlich eine Mediation zwischen Täter und Opfer statt und die Konditionen einer Rückgabe des gestohlenen Gutes werden durch die Mafia ausgehandelt. Dieser Vorgang trat besonders in Sizilien gegen Ende des 19. Jahrhunderts auf, als der Diebstahl von Vieh grassierte. Durch die Vermittlung der Mafiosi bekamen die Bestohlenen in den allermeisten Fällen einen Großteil ihrer Tiere zurück (Paoli 2003, 161). Blok (1981, 193) berichtet von einem Fall, bei dem die Rückgabe gegen eine Zahlung in Höhe von 40 Prozent des Marktwertes der Tiere erfolgte. Heute geht es anstelle der Rückgabe von Tieren in der Regel um Autos (Paoli 2003, 161).

Neben dieser Art der unparteiischen Vermittlung treten Mafiosi bei Eigentumsdelikten zum anderen als strafender Schiedsrichter auf. In diesen Fällen wird keine Einigung zwischen Dieb und Bestohlenem hergestellt, sondern das Diebesgut wird wiederbeschafft und der Dieb sanktioniert. Exemplarisch kann hierfür ein Fall aus New York stehen, bei der eine Kirche, der auch Carlo Gambino, Boss der gleichnamigen Mafiavereinigung, angehörte, Opfer von Dieben wurde. Nachdem die Polizei keinen Erfolg bei der Suche nach den sakralen Wertgegenständen hatte, wandte sich der Kirchenvorsteher an Gambino, woraufhin die Diebe schnell ermittelt und das Diebesgut wieder an 
seinen Bestimmungsort zurückgebracht wurde. Dem Priester wurde auf Nachfrage mitgeteilt, dass es nicht notwendig sei, die Polizei darüber zu informieren, da die Diebe schon genug bestraft worden seien (Davis 1994, 167).

Zusätzlich treten Mafias - entgegen der These Gambettas (1993, 167f.) - in Fällen auf, die sie nicht unmittelbar betreffen. So ermordete die Cosa Nostra einen Taschendieb, der einer tschechischen Tänzerin, die auf Tournee in Palermo war, bei einem Überfall das Gesicht verletzt hatte. Ebenso wurde der Mörder eines Jungen seinerseits ermordet, weil er das Kind nur wegen dessen zufälliger Zeugenschaft außerehelicher Liebschaften getötet hatte (Paoli 2003, 161). Ebenso erging es einem katholischen Geistlichen, der im Verdacht stand, die Dienste von Prostituierten in Anspruch zu nehmen (Paoli 2003, 163).

Mafias als „Torwächter“ - Eine letzte Drittenkonfiguration lässt sich als Zugangskontrolle zu lukrativen Geschäftsfeldern bezeichnen, legal und extra-legal. In diesen Fällen zahlen interessierte Personen nicht so sehr für ihren Schutz als vielmehr für die Erlaubnis, überhaupt eine bestimmte Tätigkeit durchführen zu dürfen (Schelling 1971). Dazu zählt auch, dass bestimmte Aktivitäten ganz untersagt werden und eben nicht ohne Schwierigkeiten gegen den Willen der jeweiligen Mafia durchgeführt werden können.

Neben der aktiven Organisation von Kartellen, wie sie Gambetta und Reuter eindrücklich theoretisieren (Gambetta/Reuter 1997), gibt es vor allem in Süditalien zahlreiche weitere Beispiele dafür, wie Mafias als „Torwächter“ operieren; beginnend damit, dass sowohl die "Ndrangheta als auch die Cosa Nostra dafür sorgt, dass auf dem Gebiet einer Familie ohne vorheriges Einverständnis keine Straftaten von Mitgliedern einer anderen Familie oder Kriminelle ohne familiäre Anbindung begangen werden dürfen. So erlaubt die 'Ndrangheta in ihrem Territorium keine kriminellen Aktivitäten ohne vorherige Autorisierung. Grundsätzlich zielen die Familien sogar darauf ab, die Verbrechensausübung zu monopolisieren und auch Kleinkriminalität zu unterbinden. Das gleiche galt lange und gilt teilweise auch heute noch für die Cosa Nostra in Sizilien, die Diebstahl und Raub zum Teil mit dem Tode bestraft. Man ist aber vielerorts stattdessen dazu übergegangen, den Kriminellen die vorherige Einholung einer Erlaubnis sowie einen Anteil an ihrer Beute abzuverlangen. Außer dieser Erlaubnis, ihrem Geschäft von der Mafia ungestört nachzugehen, erhalten diese Kleinkriminellen allerdings keine weiteren Vorteile. Schutz gegenüber rivalisierenden Gruppen oder gar gegenüber den Strafverfolgungsbehörden sind eine seltene Ausnahme (Paoli 2003, 156ff.). Das zeigt auch das 
Beispiel von neapolitanischen Camorra-Vereinigungen, die bestimmte Aktivitäten, wie illegales Glücksspiel oder den Straßenverkauf von Drogen und Zigaretten, kontrollieren und ihren Anteil kassieren, aber die konkrete Ausführung Nichtmitgliedern überlassen, die keinen nennenswerten Verlust darstellen, wenn sie der Polizei ins Netz gehen (Behan 2009, 159f.).

Anerkennungszwang und Emergenzfunktion - In der jüngeren sozialtheoretischen Forschung zur Figur des Dritten macht vor allem Gesa Lindemann (2006; 2010a; 2010b) geltend, dass Dritte in der Regel ordnungsstiftende Funktionen haben, indem sie einen „Anerkennungszwang“ vermitteln (Lindemann 2010b, 184); ein Gedanke, der sich auch wiederkehrend bei Simmel findet, ohne von ihm systematisch ausgearbeitet zu sein (Simmel 1992, 114, 126). Entweder sorgen Dritte dafür, dass ein Körper oder ein Äquivalent überhaupt als sozialer Akteur, als „Person“ gilt (konstitutive Funktion des Dritten). Oder sie garantieren bzw. regulieren den Anspruch von Personen, unter spezifischen oder diffusen sachlichen Gesichtspunkten als zwingend relevant für Andere zu gelten, so dass diese die betreffenden Personen nicht ignorieren können oder (als ganz selbstverständlich) gar nicht erst das Motiv entwickeln, die Personen zu ignorieren. Es entstehen dadurch einigermaßen beständige soziale Beziehungen, die aus Sicht eines externen Beobachters oftmals als eher unwahrscheinlich erscheinen, weil Geltungsansprüche zwischen den Beteiligten ungeklärt sind (Lindemann 2006, 98). Dritte bearbeiten diese Anerkennungsfragen (Emergenzfunktion des Dritten).

Betrachten wir die beschriebenen Drittenpositionen von Mafias - Vermittlung, Unparteilichkeit und Zugangsregulierung - unter dieser Perspektive der Ordnungsstiftung, dann laufen die jeweils beschriebenen Fälle ebenfalls häufig auf eine Produktion von Anerkennungszwängen hinaus; einschließlich von Regulierungen, in denen Ansprüche abgewiesen werden, z.B. von lokalen Kleinkriminellen (bzw. solchen, die es werden wollen), Mafias dabei also eher eine Exklusionsfunktion übernehmen. Betrachtet man die Beispiele gleichwohl auf eine Emergenzfunktion, dann zeigen sie, dass Mafias vielfältige Ansprüche durchsetzen, u.a. auf Ehre, auf Schadensersatzansprüche oder auf Partizipation an Kartellen. Die Anerkennungsfrage erscheint in dieser Sicht also als ein vielversprechender Kandidat, Mafias nicht nur numerisch, sondern auch inhaltlich präziser zu bestimmen. Dafür ist aber eine systematischere Analyse von Fällen erforderlich, als wir sie hier vorgenommen haben.

Sicher ist dagegen, dass Mafias, zumindest in den uns bekannten Fällen, kein Anerkennungsproblem haben. Alle genannten Drittenkonfigurationen sind durch die dauerhafte Anerkennung einer 
Mafia als Instanz gekennzeichnet, was sie in besonderer Weise von gewöhnlichen Kriminellen unterscheidet, die nicht auf die Etablierung dauerhafter sozialer Beziehungen zu ihren Opfern abzielen (Catanzaro 1992).[12] Mafias haben den quasi-dinglichen Charakter einer sozialen Tatsache im Sinne Émile Durkheims, die von den Einzelnen anerkannt werden muss; ihre Position als Dritter „becomes a widely known fact“ (Behan 2009, 158).

Wenn man weiter bedenkt, dass Mafias als Element nicht in einer Dreierkonfiguration aufgehen, sondern lokal in vielfältiger Weise als Dritte fungieren, dann entstehen die Voraussetzungen, Dritte sein zu können, vermutlich nicht aus einer konkreten Konfiguration mit Anderen, sondern vielmehr aus der Art des „bridging“ (Martin 2009, 30) zwischen der Beteiligung an vielen Konfigurationen, einschließlich legaler oder extra-legaler Zweierbeziehungen. Der Schlüssel zum Verständnis (a) von Mafias als anerkanntem Dritten in konkreten Konfigurationen mit Anderen sowie (b) der Verknüpfung diverser Beteiligungen als Dritte (und Zweite) liegt aus unserer Sicht in ihrer formalen Organisation. Mafias sind organisierte Dritte.

\section{Mafias als organisierte Dritte}

Die Etablierung einer Mafia als Dritter ist kein zwangloser Prozess. Vielmehr beruht ihre dauerhafte Anerkennung als Dritter und um Anerkennungszwänge zu erzeugen auf ihrem Droh- und Gewaltpotential. Selbst in Fällen, in denen es ihnen gelingt, die Interaktionen für die Partner angenehm und vorteilhaft zu gestalten, bleibt die Möglichkeit, Gewalt einzusetzen, hintergründig präsent und letzte Grundlage der Anerkennung der jeweiligen Mafia (P. Schneider/J. Schneider 2003, 118f.; Paoli 2003, 96, 102; Catanzaro 1992).

Das Gewaltpotential von Mafias drückt sich nicht nur quantitativ in der Fähigkeit aus, eine überlegene Menge gewaltbereiter Personen mobilisieren zu können, sondern auch qualitativ darin, dass es sich bei diesen Personen um erprobte und routinierte Gewalttäter handelt (Collins 2008, 370ff.). Der Einzelne sieht sich damit einer Mobilisierbarkeit von (Gewalt-)Ressourcen gegenüber, denen er typischerweise unterlegen ist, eine Asymmetrie, die James Coleman bekanntermaßen als typisch für das Verhältnis von natürlichen Personen und korporativen Akteuren betrachtet (Coleman 1986, 34). Einzelpersonen, die Beziehungen zu Mafias unterhalten, wissen um diese Asymmetrie, wie eine Geschichte über den Besitzer der größten Supermarktkette Palermos verdeutlicht, die sich in der
[12] Siehe dazu auch das instruktive Beispiel der „Schwarzen Hand“ in New York Anfang des 20. Jahrhunderts bei Dash 2010, 86ff. 
Stadt erzählt wird. Er habe, nachdem seine bisherigen ,Schutzherren` ins Gefängnis gewandert sind, verzweifelt nach einem neuen Kontakt zur Cosa Nostra gesucht, um seinen pizzo zu entrichten (LaRosa 2012, 39)[13]. Mag die Geschichte nun stimmen oder nicht, sie lässt erahnen, worauf die Anerkennung der Cosa Nostra beruht. Sie basiert auf der Erwartung, dass selbst dann, wenn die konkrete natürliche Person, die sich ihre ,Freundschaft' hat bezahlen lassen, nicht mehr existiert, eine ,Familie', die hinter dem ,Freund‘ steht, gleichwohl besteht - oder aber deren Ansprüche von einem anderen Clan, der an ihre Stelle getreten ist, geltend gemacht werden.

Für Mafias ist es demgegenüber egal, ob sie von einem bestimmten Geschäftsmann als Person Schutzgeld kassiert oder nicht. Wichtig ist nur, dass sie Schutzgelder erhält. Somit ist eine Eliminierung des Geschäftsmanns nicht von Belang, da Alternativen bereitstehen. Natürlich besteht von Seiten des Geschäftsmannes immer die Möglichkeit die Strafverfolgungsbehörden zu informieren. Aber diese können nicht jeden Einzelnen permanent schützen.

Das kollektive Mobilisierungspotential von Mafias zeigt sich ebenfalls in ihrem Verhältnis zu Strafverfolgungsbehörden. Denn die zuvor erwähnten Asymmetrien können sich nicht nur zwischen natürlichen und korporativen Akteuren, sondern auch im Verhältnis zwischen Korporationen ergeben. Was die rein quantitative Mobilisierungsfähigkeit angeht, sind die Strafverfolgungsbehörden einer Mafia natürlich heillos überlegen. Allerdings begeben sich Mafias in der Regel nicht in einen offenen Konflikt mit der Gesamtheit der Strafverfolgungsbehörden, wodurch dieser Vorteil nivelliert wird. Die starke Geheimhaltung, eine fehlende Bindung an Gesetze und ihre Informationsnetzwerke können Mafiosi zudem Vorteile gegenüber den Ermittlungsbeamten verschaffen (I.M.D./Catalano 2011, 186ff.). Außerdem ermitteln in den meisten Fällen nur einige Beamte, die dann als Person konkret gefährdet sind, was Asymmetrien gegenüber einer Mafia bedingen kann. Die bessere finanzielle Ausstattung von Mafias, die das Gehalt einzelner Beamter übersteigt, bietet zudem Möglichkeiten der Korruption und des Erwerbs ausgefeilter technischer Hilfsmittel. Demgegenüber sind die Ermittlungsbeamten nicht immer aufs Beste ausgerüstet und erfahren auch in ihren eigenen Behörden aus diversen Gründen mitunter wenig Unterstützung, wenn sie gegen Mafias vorgehen. Einige sizilianische Polizisten sahen sich bei ihren Ermittlungen gegen die Cosa Nostra sogar gezwungen, ihre Privatfahrzeuge zu verwenden und den Sprit selbst zu bezahlen (Battaglia/Orlando 2008, 167).

Alles in allem sind Mafias folglich (und wie Kriminalität es meistens zu sein pflegt; Gambetta 2009, 3) kollektive Unternehmungen, die darüber hinaus besonders personalintensiv sind (Gam-
[13] Pizzo bezeichnet auf Sizilien den Tribut, den Geschäftsleute an die lokalen Mafias zahlen. 
betta 1993, 59). Die Frage, wer überhaupt Mitglied sein kann, wird dabei gleichwohl sehr sensibel behandelt, nicht zuletzt weil in der „Unterwelt“ ein Mangel an Vertrauen herrscht, der nicht durch staatliche Gesetze und Instanzen kompensiert werden kann (Gambetta 2009). Es ist daher mit erheblichen Hürden und jahrelangen Anwartschaften verbunden, in die Kreise von Mafias aufgenommen zu werden. Mafias sind sozial gesehen äußerst exklusiv. Sie erreichen diese Exklusivität durch formale Organisation.

Wenn man mit dem Begriff Organisation jedes planvolle, kollektive Handeln bezeichnet, dann sind Bankräuber, Fälscherbanden und Taschendiebe genauso organisiert wie die bisher diskutierten Mafias. Wir wollen den Begriff der Organisation dagegen in einem soziologisch präziseren Verständnis als formale Organisation fassen. Das Alleinstellungsmerkmal formaler Organisationen gegenüber sämtlichen anderen Formen sozialer Organisation liegt darin, dass sie - als ein besonderer Typ sozialer Systeme (Luhmann 1975) - den Beitritt unter die Bedingung der Akzeptanz von Mitgliedschaftserwartungen stellen. Formalität ist dabei eine Qualität dieser Erwartungen, insofern ihre Erfüllung Bedingung der Mitgliedschaft ist. Wer diese Erwartungen nicht akzeptiert und durch sein laufendes Verhalten affirmiert, kann nicht Mitglied in der Organisation werden bzw. bleiben (Luhmann 1964, 29ff.).[14] Die Akzeptanz dieser Mitgliedschaftsbedingungen wird durch die Beitrittsentscheidung ausdrücklich kommuniziert (Luhmann 1964, 40).

Natürlich sind Mafias keine Verwaltungsorganisationen, aber grundsätzlich funktioniert der Mitgliedschaftsmechanismus bei ihnen genauso wie bei legalen Organisationen. Anders als bei ,natürlichen`Familien gehören Mitglieder nicht alternativlos zum System, was eine Beitrittsentscheidung erforderlich macht, die klar erkennbar mitteilt, dass man bereit ist, die Präferenzen der Organisation zu akzeptieren. Daher heben Mafias den Organisationsbeitritt, funktional äquivalent zu den Arbeitsverträgen und Mitgliedsanträgen legaler Organisationen, durch besondere Verfahren oder Übergangsriten hervor (z.B. Davis 1994, 23f.; Paoli 2003, 67f.; Hill 2006, 67). Dadurch entsteht eine Selbstverpflichtung des konkreten Mitglieds, an die von anderen Mitgliedern erinnert werden kann, sollte es notwendig werden. Es stört nämlich die Konsistenz der individuellen Selbstdarstellung, wenn man gegen eine Ordnung verstößt, für deren Anerkennung man sich bewusst und öffentlich sichtbar entschieden hat (Luhmann 1964, 37). Dieser Mitgliedschaftsmechanismus ermöglicht eine Generalisierung der Motivlage der Mitglieder und in der Folge die Institutionalisierung von zum Teil außergewöhnlichen, im Fall von Mafias sogar illegalen Verhaltensweisen. [15]
[14] Dabei ist die schriftliche Formulierung einer Erwartung nicht Bedingung für ihre Formalisierung. Schriftlichkeit kann zwar eine solche Erwartung indizieren, jedoch genügt es, wenn die Erwartung „definierbar“ ist (Luhmann 1969, 393). Mündlich ausgesprochene Erwartungen können also, als ungeschriebene Gesetze, ebenso als Mitgliedschaftsbedingung gelten.

[15] Dazu formuliert Luhmann prägnant: „Die Soldaten marschieren, die Schreiber protokollieren, die Minister regieren - ob es ihnen in der Situation nun gefällt oder nicht (Luhmann 1975, 12). 
Mitunter wird Mafias abgesprochen, formale Organisationen zu sein, da der Austritt oftmals mit hohen Kosten verbunden ist und z.B. nur durch den Tod des Mitglieds erfolgen kann (Paul/Schwalb 2011, 338; Pohlmann/Markova 2011, 22ff.). Für das einzelne Mitglied mag die Form des Austritts einen gravierenden Unterschied machen, für die Formalisierung von Erwartungen ist die Freiwilligkeit oder Leichtigkeit des Austritts irrelevant. Die Mitgliedschaft muss lediglich durch Entscheidung veränderbar sein, d.h. Nichtmitgliedschaft muss eine mögliche Alternative sein. Entscheidend ist also die Kontingenz der Mitgliedschaft, die auch bei Mafias gegeben ist und zwar nicht nur in Form von Tod, sondern z.B. auch durch staatliche Zeugenschutzprogramme. Im Übrigen besteht hier eine Parallele von Mafias zu Staaten, insofern auch das staatliche Recht auf der Möglichkeit des gewaltsamen Entzugs von Bürgerrechten beruht, sei es durch Haft oder Tod (Luhmann 1964, 44f.).

Die Mitgliedschaftserwartungen von Mafias sind sehr umfassend und reichen bis in das Privatleben der Mitglieder. Sie beziehen sich dabei (neben der Bereitschaft zu Kriminalität) aber vor allem - direkt oder indirekt - auf die Notwendigkeit der Geheimhaltung ihrer Aktivitäten, womit sie sich nur graduell, nicht aber grundsätzlich von anderen Organisationen unterscheiden (Westerbarkey 1998, 152f.; Weber 1976). Trotz dieser weitreichenden Erwartungen findet aber bei Mafias keine Totalinklusion der Mitglieder statt und sie entsprechen auch nicht dem Typus vorneuzeitlicher Korporationen, die aus Personen anstatt aus Positionen bestehen (Coleman 1986, 169f.). Mafias sind keine Alternative zum Leben in Familienhaushalten. Auch bei ihnen kennen die Mitgliedschaftserwartungen Grenzen. Beispielsweise findet kein Eingriff in die legalen wirtschaftlichen Aktivitäten der Mitglieder statt und es steht ihnen oftmals frei eigenständige illegale Unternehmungen mit anderen Kriminellen durchzuführen (Arlacchi 1993, 74; Paoli 2003, 145ff.).

$\mathrm{Zu}$ den Erwartungen, auf die sich Mafiosi verpflichten, gehört auch eine unbedingte Gewaltbereitschaft. Für Exekutionskommandos finden sich in der Regel leicht einige Kombattanten (Arlacchi 1993, 92ff.). Mafias erreichen damit die bereits skizzierte Mobilisierungsfähigkeit, die Gewalttätigkeit einschließt (siehe dazu auch Paul/Schwalb 2011, 135). Insbesondere durch ihre hohe Exklusivität können ihre Mitglieder untereinander - und unabhängig von den Beitrittsmotiven des Einzelnen - mit einem hohen Maß an erforderlicher Konformität rechnen.

Neben ihrer Mobilisierungsfähigkeit entschärfen Mafias durch ihre Formalität ein Stück weit das Vertrauensproblem zwischen den Mitgliedern, das sich oftmals gerade daraus ergibt, dass Mafiosi als konkrete Dritte fungieren.[16] Deswegen existiert immer die Möglichkeit, dass sie, womöglich
[16] In diesem Auftreten unterscheiden sich die Mafiosi von anonymen Dritten, die, folgt man einem theoretischen Argument Niklas Luhmanns, kontextunabhängige soziale Ordnungen stiften können (Luhmann 1972, 66) 
„schwankend und beeinflussbar“ (Luhmann 1972, 66) im persönlichen Kontakt, durch die anderen Parteien korrumpiert werden oder als so genannte Grenzstellen der Organisation (Luhmann 1964, 220ff.) Erträge in die eigene Tasche abzweigen. Durch eine selektive Personalauswahl und der immer wieder erneuerten Gewalt- oder Ausschlussdrohung auch gegenüber Mitgliedern bauen Mafias der Ausnutzung dieser Gelegenheiten vor (ohne sie natürlich restlos verhindern zu können). Mafiosi, die ihre Selbstverpflichtung gegenüber, ihrer ' Organisation vernachlässigen würden, setzen das in sie gesetzte Vertrauen aufs Spiel. Dieser generelle Vertrauensakt von Seiten der anderen Mitglieder oder eines repräsentativen Gremiums funktioniert hier wie eine „Fessel“ (Luhmann 2000, 78ff.). In dieser Perspektive sind Mafias als organisierte Dritte taktisch konzipiert, und nicht etwa auf Basis von geteilten Gesinnungen, die nicht zuletzt leicht zu imitieren sind (Gambetta 2009, 174ff.).

\section{Fazit und Forschungsperspektiven}

Die an Simmel angelehnte numerische Perspektive erlaubt es uns, die Vielfalt der Aktivitäten von Cosa Nostra, 'Ndrangheta, US-amerikanischen Familien und der Yakuza abstrahierend als Drittenkonfigurationen zu betrachten, in denen sich zahlreiche „Materien der Vergesellschaftung“ realisieren können (Simmel 1992, 18). Vermittlung, Unparteilichkeit und Zugangsregulierung sind dabei drei mögliche, aber sicherlich nicht alle denkbaren Positionen, in denen Mafias soziale Beziehungen zwischen Zweien oder Mehreren (mit-)gestalten, indem sie, so unserer vorläufiges Argument, für eine Anerkennung von Ansprüchen einer oder mehrerer Parteien sorgen; und nicht primär für Schutz.

Als vorläufig betrachten wir es vor allem deswegen, weil die von uns diskutierten Fälle zwar in diese Richtung deuten, ihre Zusammenstellung jedoch nicht den systematischen Anforderungen komparativer Forschung genügt. Darin sehen wir ein zentrales Desiderat unserer Studie, ebenso in der systematischen numerischen Betrachtung der vielfältigen Beziehungsmuster zwischen Mafias und staatlichen Stellen, die wir für den Beitrag zunächst einmal zurückgestellt haben (siehe dazu die abschließende Passage der Einleitung).

Ein drittes Desiderat hängt eng mit dem Anerkennungsproblem zusammen, das sich für Mafias selbst stellt. Dass ihre Mitglieder tatsächlich als Instanzen der Vermittlung, der Mediation oder der Zugangskontrolle anerkannt werden, sehen wir in der formalen Organisiertheit von Mafias be- 
gründet. Ihre starke formal vermittelte Exklusivität erklärt zum einen ihre Mobilisierungsfähigkeit gegen jeden, der ihre Position infrage stellt. Zum anderen schwächt Formalität das grundsätzliche Vertrauensproblem in extra-legalen Unternehmungen ab. Dieser Zusammenhang von Drittenposition und formaler Organisiertheit führt zu der Frage, welche weiteren organisierten Dritten es neben Mafias gibt. Staatliche Ordnungsbehörden sind sicherlich naheliegende Kandidaten. Ein sowohl konfigurationaler als auch äquivalenzfunktionalistischer Vergleich zwischen Polizeien, Armeen und Mafias erscheint daher besonders lohnenswert.

Trotz ihrer Vorläufigkeit sehen wir die These, Mafias seien organisierte Dritte, als Beitrag zu gleich zwei aktuellen, bisher jedoch nicht miteinander koordinierten Debatten in der soziologischen Forschung. Erstens richtet er sich an die gegenwärtig an der Figur des Dritten orientierte Grundlagenforschung, in der organisationssoziologische Argumente marginalisiert sind, aus unserer Sicht jedoch zum Verständnis beitragen, wie konkrete Dritte eigentlich dauerhaft ihre Position gegenüber anderen Parteien sichern. Zweitens adressieren wir die soziologisch-kriminologische Forschung zu organisierter Kriminalität, in der als offen gelten kann, was das Attribut der Organisiertheit eigentlich bezeichnet. Wir sehen die These deswegen auch als Vorschlag, die Einsichten der Theorie formaler Organisationen im Anschluss an Chester Barnard (1968), Niklas Luhmann (1964) oder jüngst Arthur Stinchcombe (2001) fruchtbar zu machen, um Phänomene der Koordination, Kohäsion und Selbstverpflichtung von Kriminellen besser zu verstehen.

\section{Bibliographie}

Albini, J. (1971) The American Mafia: Genesis of a Legend. New York: Appleton-Century-Crofts. Arlacchi, P. (1993) Mafia von innen. Das Leben des Don Antonio Calderone. Frankfurt am Main: S.

Fischer.

Barnard, C. (1968) The Functions of the Executive. London: Oxford University Press.

Battaglia, P./Orlando, L. (2008) Leoluca Orlando erzählt die Mafia. Freiburg: Herder.

Behan, T. (2009) See Naples and Die: The Camorra and Organized Crime. London: Tauris.

Blok, A. (1981) Die Mafia in einem sizilianischen Dorf 1860-196o. Eine Studie über gewalttätige 
bäuerliche Unternehmer. Frankfurt am Main: Suhrkamp.

Castells, M. (2010) End of Millennium: The Information Age: Economy, Society, and Culture III.

Chichester: John Wiley \& Sons.

Catanzaro, R. (1985) Enforcers, Entrepreneurs, and Survivors: How the Mafia Has Adapted to

Change. In: British Journal of Sociology 36(1): 34-57.

Catanzaro, R. (1992) Men of Respect. A Social History of the Sicilian Mafia. New York: The Free Press.

Chu, Y. (2000) The Triads as Business. London: Routledge.

Chubb, J. (1996) The Mafia, the Market and the State in Italy and Russia. In: Journal of Modern

Italian Studies 1(2): 273-291.

Coleman, J. (1986) Die asymmetrische Gesellschaft. Weinheim; Basel: Beltz.

Collins, R. (2008) Violence. A Micro-Sociological Theory. Princeton: Princeton University Press.

Cressey, D. (1969) Theft of the Nation: The Structure and Operations of Organized Crime in America. New York: Harper \& Row.

Dash, M. (2010) The First Family. Terror, Extortion, Revenge, Murder, and the Birth of the American Mafia. New York: Ballantine Books.

Davis, J. (1994) Mafia Dynasty: The Rise and Fall of the Gambino Crime Family. New York: HarperCollins.

Dickie, J. (2007) Cosa Nostra. Die Geschichte der Mafia. Erw. Ausg., Frankfurt am Main: Fischer Taschenbuch Verlag.

Dietz, G. (2011) Die 'Ndrangheta. Der geheime Aufstieg der kalabrischen Mafia. Weinheim: Wiley-VCH.

Fischer, J. (2008) Tertiarität. Die Sozialtheorie des „Dritten“ als Grundlegung der Kultur- und Sozialwissenschaften. In: Raab, J. et al., (Hg.) Phänomenologie und Soziologie. Wiesbaden: VS Verlag für Sozialwissenschaften.

Freund, J. (1976) Der Dritte in Simmels Soziologie. In: Böhringer, H./Gründer, K. (Hg.) Ästhetik und Soziologie um der Jahrhundertwende: Georg Simmel. Frankfurt am Main: Vittorio Klostermann.

Gambetta, D. (1993) The Sicilian Mafia. The Business of Private Protection. Cambridge: Harvard University Press.

Gambetta, D. (2009) Codes of the Underworld: How Criminals Communicate. Princeton: Princeton University Press. 
Gambetta, D. (2011) „The Sicilian Mafia“ Twenty Years After Publication. In: Sociologica 5(2): 1-11.

Gambetta, D./Reuter, P. (1997) Conspiracy Among the Many: The Mafia in Legitimate Industries. In: Fiorentini, G./Peltzman, S. (eds.) The Economics of Organised Crime. Cambridge: Cambridge University Press.

Giordano, C. (1994) Der Ehrkomplex im Mittelmeerraum: Sozialanthropologische Konstruktion oder Grundstruktur mediterraner Lebensformen. In: Vogt, L./Zingerle, A. (Hg.) Ehre. Archaische Momente in der Moderne. Frankfurt am Main: Suhrkamp.

Haller, M. (1992) Bureaucracy and the Mafia: An Alternative View. In: Journal of Contemporary Criminal Justice 8(1): 1-10.

Hessinger, P. (2002) Mafia und Mafiakapitalismus als totales soziales Phänomen. In: Leviathan 30(4): 482-508.

Hill, P. (2006) The Japanese Mafia. Yakuza, Law, and the State. Oxford: Oxford University Press.

Hughes, E. (1971) Going Concerns: The Study of American Institutions. In: The Sociological Eye: Selected Papers. Chicago; New York: Aldine Atherton.

I.M.D./Catalano, R. (2011) 100 \% Bulle. Aus dem Alltag eines Mafia-Fahnders. München: Droemer.

Lakatos, I. (1970) Falsification and the Methodology of Scientific Research Programmes. In: Lakatos, I./Musgrave, A. (eds.) Criticism and the Growth of Knowledge. London: Cambridge University Press.

LaRosa, L. (2012) Tschüss, Mafia. In: chrismon (09.2012): 36-41.

Lindemann, G. (2006) Die Emergenzfunktion und die konstitutive Funktion des Dritten. Perspektiven einer kritisch-systematischen Theorieentwicklung. In: Zeitschrift für Soziologie 35(2): 82-101.

Lindemann, G. (2010a) Die Emergenzfunktion des Dritten - ihre Bedeutung für die Analyse der Ordnung einer funktional differenzierten Gesellschaft. In: Zeitschrift für Soziologie 39(6): 493-511.

Lindemann, G. (2010b) Statt der Dyade: die Ego-Alter-Tertius-Konstellation als konstitutive Bedingung von Sozialität. In: Bedorf, T./Fischer, J./Lindemann, G. (Hg.) Theorien des Dritten. Innovationen in Soziologie und Sozialphilosophie. München: Fink.

Luhmann, N. (1964) Funktionen und Folgen formaler Organisation. Berlin: Duncker \& Humblot. 
Luhmann, N. (1969) Gesellschaftliche Organisation. In: Ellwein, T. u. a. (Hg.) Erziehungswissenschaftliches Handbuch, Bd. 1. Berlin: Rembrandt.

Luhmann, N. (1972) Rechtssoziologie, 2 Bände. Reinbek bei Hamburg: Rowohlt.

Luhmann, N. (1975) Interaktion, Organisation, Gesellschaft. In: Soziologische Aufklärung 2. Aufsätze zur Theorie der Gesellschaft. Opladen: Westdeutscher Verlag.

Luhmann, N. (1992) Die Wissenschaft der Gesellschaft. Frankfurt am Main: Suhrkamp.

Luhmann, N. (2000) Vertrauen. Ein Mechanismus der Reduktion sozialer Komplexität. 4. Aufl., Stuttgart: Lucius \& Lucius.

Lupo, S. (2002) Die Geschichte der Mafia. Düsseldorf: Patmos.

Martin, J. (2009) Social Structures. Princeton: Princeton University Press.

Nelken, D. (1995) Review: The Sicilian Mafia: The Business of Private Protection, by Diego Gambetta. In: British Journal of Criminology 35: 287.

Paoli, L. (1999a) Die italienische Mafia: Paradigma oder Spezialfall der organisierten Kriminalität? In: Monatsschrift für Kriminologie und Strafrechtsreform 6: 425-440.

Paoli, L. (1999b) The Political-Criminal Nexus in Italy. In: Trends in Organized Crime 5(2): 15-58.

Paoli, L. (2003) Mafia Brotherhoods. Organized Crime, Italian Style. Oxford: Oxford University Press.

Paul, A./Schwalb, B. (2011) Wie organisiert ist das organisierte Verbrechen? In: Leviathan 39(1): 125-140.

Pohlmann, M./Markova, H. (2011) Soziologie der Organisation Konstanz: UVK.

Popitz, H. (2010) Vorlesung 1. Einleitung: Grundlegende Fragestellungen der Soziologie: Fünf Problemkreise. In: Einführung in die Soziologie. Konstanz: Konstanz University Press.

Savona, E. (2012) Italian Mafias' Asymmetries. In: Siegel, D./van de Bunt, H. (eds.) Traditional Organized Crime in the Modern World. New York: Springer.

Schelling, T. (1971) What is the Business of Organized Crime? In: Journal of Public Law 20: 71-84. Schneider, J./Schneider, P. (2004) Mafias. In: Nugent, D./Vincent, J. (eds.) A Companion to the Anthropology of Politics. Malden: Wiley-Blackwell.

Schneider, P. (1994) A Review of Diego Gambetta, The Sicilian Mafia. The Business of Protection. In: Italian Politics and Society Newsletter 42: 26-28.

Schneider, P./Schneider, J. (2003) Reversible Destiny: Mafia, Antimafia, and the Struggle for 
Palermo. Berkeley; Los Angeles: University of California Press.

Simmel, G. (1992) Soziologie. Untersuchungen über die Formen der Vergesellschaftung. Frankfurt am Main: Suhrkamp.

Smith, D. (1976) Mafia: The Prototypical Alien Conspiracy. In: The ANNALS of the American Academy of Political and Social Science 423(1): 75-88.

Stinchcombe, A. (2001) When Formality Works. Authority and Abstraction in Law and Organizations. Chicago: University of Chicago Press.

Superti, C. (2009) Addio Pizzo: Can a Label Defeat the Mafia? In: The Journal of International Policy Solutions 11(Spring): 3-11.

Tyrell, H. (2008) Zwischen Interaktion und Organisation: Gruppe als Systemtyp. In: Heintz, B. et al (Hg.) Soziale und gesellschaftliche Differenzierung. Wiesbaden: VS Verlag für Sozialwissenschaften.

Tzvetkova, M. (2008) Aspects of the Evolution of Extra-legal Protection in Bulgaria (1989-1999). In: Trends in Organized Crime 11(4): 326-351.

Vaccaro, A. (2012) To Pay or Not to Pay? Dynamic Transparency and the Fight Against the Mafia's Extortionists. In: Journal of Business Ethics 106(1): 23-35.

Varese, F. (2005) The Russian Mafia. Private Protection in a New Market Economy. Oxford: Oxford University Press.

Varese, F. (2011) Mafias on the Move. How Organized Crime Conquers New Territories. Princeton: Princeton University Press.

Varese, F. (2005) The Russian Mafia. Private Protection in a New Market Economy. Oxford: Oxford University Press.

Weber, M. (1976) Wirtschaft und Gesellschaft. Tübingen: Mohr Siebeck.

Westerbarkey, J. (1998) Das Geheimnis. Die Faszination des Verborgenen. Leipzig: Gustav Kiepenheuer.

White, H. (2008) Identity and Control. How Social Formations Emerge. Princeton: Princeton University Press.

Ziemann, A. (2011) Die Zahl als soziologische Kategorie und die Quantität sozialer Verhältnisse. In: Tyrell, H./Rammstedt, O./Meyer, I. (Hg.) Georg Simmels große „Soziologie“. Eine kritische Sichtung nach hundert Jahren. Bielefeld: Transcript. 